\title{
Téoros
}

Revue de recherche en tourisme

\section{Parcs de loisirs et patrimoine religieux}

\section{André Hut}

Volume 12, numéro 3, octobre 1993

URI : https://id.erudit.org/iderudit/1077940ar

DOI : https://doi.org/10.7202/1077940ar

Aller au sommaire du numéro

\section{Éditeur(s)}

Université du Québec à Montréal

\section{ISSN}

0712-8657 (imprimé)

1923-2705 (numérique)

Découvrir la revue

Citer cet article

Hut, A. (1993). Parcs de loisirs et patrimoine religieux. Téoros, 12(3), 47-49.

https://doi.org/10.7202/1077940ar d'utilisation que vous pouvez consulter en ligne.

https://apropos.erudit.org/fr/usagers/politique-dutilisation/ 


\title{
EU R-INE $O$ Parcs de loisirs et patrimoine religieux
}

\author{
André Hut ${ }^{*}$
}

Les parcs de loisirs sont concernés à divers titres par le patrimoine religieux et il n'est nullement saugrenu de citer celui-ci comme un des thèmes privilégié et incontournable de ce tourisme culturel: à travers les parcs patrimoniaux d'une part et leur interprétation culturelle et spirituelle d'autre part.

\section{Parcs patrimoniaux}

À la demande circonstanciée des visiteurs, les parcs naturels et culturels d'origine religieuse offrent une réponse de qualité; de plus, les parcs thématiques héritent bénéfiquement desracines culturellesissues du patrimoine religieux.

\section{Motivations}

Commel'évoquait déjà, en 1985, un article précédent de Téoros ${ }^{(1)}$ sur les nouvelles sensibilités culturelles, deux courants se développent parallèlement aujourd'hui et sont destinésà se rencontrer et se renforcer mutuellement.

D'une part, de plus en plus de visiteurs, résidents et touristes, s'intéressent aux patrimoines et phénomènes religieux: partout et en nombre croissant, ils se présentent dans desabbayes, églises historiques et musées à contenu religieux.

D'ailleurs, de tout temps et plusque jamais, les êtres humains n'ont-ils pas cherché des réponses satisfaisantes aux questions fondamentales et existentielles qu'ils se posent: D'où venons-nous? Où allons-nous? Pourquoi la mort? Qu'y a-t-il après?' Quel est l'avenir de la planète?

En outre, dans un monde de mobilité et de déracinement, ne sont-ils pas en quête aussi de leurs racines sociales, culturelles et spirituelles?

Monsieur Andre Hut de Bruxelles, Belgique, est joumaliste, formateur et expert-conseil en animation touristique.

\section{Parcs naturels et culturels}

Parmi les parcs paysagers, il faut compter un nombre important d'abbayes, couvents, béguinages et institutions caritatives religieuses, presbytères... qui ont développé des jardins d'agrément et des cultures de plantes médicinales ${ }^{(t)}$. En outre, on assiste actuellement à une redécouverte émerveillée des grands cimetières urbains, comme celui du Père Lachaise, à Paris, qui offrent, en pleine ville, des aires deverdure et de calme, des invitations à un pèlerinage culturel à la recherche de personnages célèbres qui y sont enterrés ainsi que l'admiration de chefs-d'oeuvres réalisés par des artistes sculpteurs de réputation ${ }^{(j)}$. Certaines abbayes en ruines, détruites par les guerres et la Révolution française, sont reconnues et classées tout à la fois comme réserves naturelles, refuges pour une faune et une flore exceptionnelles, centres de rencontres et d'animation culturelles, base d'interprétation de l'architecture et de l'expérience monastiques.

Ils font partie, les uns et les autres, de cet hếritage patrimonial que $\mathrm{la}$ Charte de Florence (1981), élaborée par le Comité International des Jardins Historiques, veut sauvegarder et mettre en valeur. C'est pourquoi la Commission des Communautés Européennes, décidant de poursuivre son action pour la dixième année consécutive en faveur du patrimoine architectural et d'organiser, depuis 1988 , un concours centrés sur des thèmes annuels, a choisi les jardins historiques considérés comme des monuments, pour l'année $1993^{(4)}$.

\section{Racines culturelles}

Il est impossible de visiter la Cité des Sciences et de l'Industrie de La Villette, à Paris, ou le Futuroscope de Poitiers consacré à l'image et à la communication sans évoquer les contributions créatrices qu'ont apportées ces hommes et ces femmes des monastères comme autant d'antécédents irréversibles à l'histoire culturelle, artistique et scientifique des pays occidentaux.
Le réseau des abbayes cisterciennes notamment et les routes de pèlerinage vers Compostelle, en Espagne, illustrent tellement les expériences fondatrices d'échanges, d'hospitalité et de guidage à la base des voyages et de l'animation touristiques d'aujourd'hui que le Conseil de l'Europe a élaboré des itinéraires culturels européens afin de les valoriser.

$\mathrm{Nul}$ ne pourra contester que les personnages historiques ou légendaires qui orchestrent l'animation de certains parcs d'attraction n'aient été véhiculéset diffusés par les saltimbanques, au pied des églises, à l'occasion des fêtes foraines - kermesses ou autres - commémorant l'anniversaire de la construction de celles-ciou la consécration au saint patron tutélaire auquel elles sont dédiées ${ }^{(5)}$. Ainsi, à titre d'exemples, Guillaume Tell et le village d'Heidi en Suisse; Astérix, les Schtroumphs ou le Fantasyland d'EuroDisney en France, Tintin a Walibi en Belgique ou De Efteling en Hollande ${ }^{(6)}$.

De plus, les enluminures des incunables, les sculptures des chapiteaux au sommet des colonnes ou des tympans à la porte des cathédrales, les rétables et les peintures en triptyques, les feuilletons biographiques décorant les chầsses... ne constituent-ills pas des références techniques et méthodologiques aux récits *en images que popularisent de même aujourd'hui les bandes dessinées évoquées ci-dessus?

\section{Clefs d'interprétation}

L'action pédagogique des musées à contenu religieux n'est-elle pas destinée justement à donner aux visiteurs les clefs d'interprétation culturelle et spirituelle du patrimoine religieux ${ }^{2()}$ En fait, il existe plus d'une cinquantaine de musées de divers types en Communauté française de Belgique, offrant exclusivement ou partiellement à la découverte des objets, activités et traditions ayant trait aux phénomènes spirituels ainsi qu'aux comportements individuels ou aux manifestations collectives concernant des religions de divers peuples etcivilisations. Il en va de mêmedes trésors 
existants dans les églises ou communautés religieuses.

C'est pourquoi l'Association *Animation Chrétienne en Tourisme (ACT) a organisế, pour la deuxième fois, un colloque sur le sujet dans le cadre du Salon des vacances de Bruxelles, le 31 mars 1993. L'ACT y a invité les responsables de ces musées spécifiques à rencontrer d'autres collègues, chargés comme eux des fonctions de communication, d'animationet d'éducation visà-vis de visiteurs individuels ou en groupes. Elle leur proposait de partager ensemble les options et réalisations - muséologiques et pédagogiques - qui permettent à chacun de répondre aux centres d'intérêts culturels, à la curiosité intellectuelle et aux interrogations spirituelles des personnes qui se présentent chez eux.

Les visiteurs ne s'attendent-ils pas à un accueil et une animation de qualité? Ils ont droit, en effet, à comprendre la signification de ces manifestations religieuses resituées dans leur contexte historique, culturel et spirituel.

\section{Le colloque de I'Association ACT}

Le programme annoncé du colloque comprenait deux parties amorçant le débat.

La première se composait de trois exposés introductifs, plus généraux. D'abord, la communication demonsieur J. Liébin: *La philosophie et la pratique des écomusées»; en effet, l'écomusée favorise la redécouverte et la réappropriation par une population locale de ses divers patrimoines - y compris le religieux - dont elle hérite de ses prédécesseurs. Les objets présentés dans les musées à contenu religieux remplissent toujours une fonction en référence aux cérémonies culturelles d'une communauté précise ou à la pratique individuelle d'un rite manifestantl'appartenance à celle-ci et l'adhésion à ses croyances.

Ensuite, madame C. Deltour des Musées Royaux d'Art et d'Histoire était sollicitée pour expliciter le rồle, les démarches et les outils par lesquels un musée révèle aux visiteurs la signification ultime des objets contribuant à définir et renforcer l'identité collective d'une communauté humaine dont le sacré et la religion en sont une des composantes fondamentales et individuantes.
Enfin, le signataire André Hut, animateur culturel, chargé de la conception du programme, avait à dresser un inventaire des besoins, aspirations et attentes, conscientes ou non, qui habitent les visiteurs à leur arrivée.

La deuxième partie était destinée à compléter les considérations plus générales des exposés introductifs parlarelation de quatre expérimentations provenant respectivement d'une province différente et qui sont explicitées ci-dessous.

\section{Réamorcer le dialogue}

Comme si les objets étaient des princesses endormies n'attendant que le baiser du prince charmant pour «renaître à la vies et à l'amour: voilà ce qu'ont démontré les divers orateurs! L'allusion aux contes de fées n'est pas innocenté et une psychanalyse de ceux-ci qu'en a faite Bruno Bettelheim n'est pas étrangère à la démarche muséale ${ }^{(i)}$.

En effet, Claudine Deltour a rappelé que l'objet a *une histoire à raconter, son histoires. Qu'il faut le réveiller de son sommeil et lui rendre la parole. *Ce qui importe, c'est de passer del'objet-témoin à l'objet-dialogue. Ce dialogue portera tout naturellement sur lemessage de l'objet... et sera fonction de l'objets.

André Neuberg du Musée de Piconrue, à Bastogne, confirme qu'il est *important de remettre le patrimoine religieux dans le cadre de vie quotidien des gens pour lequel ces oeuvres ont été faites et qui leur donne un sens, "vie et raison d'être" $\$$.

À son tour, ce qui frappe Suzanne Vandecandu Trésor d'Hugode Oignies, à Namur, *c'est de surprendre et de suivre l'attrait spirituel qu'un chacun découvre face à des oeuvres d'art qui, tout en révélant le don exceptionnel de l'artiste, orientent d'une façon surprenante dans une contemplation inattendue toute intérieure qui meut et bouleverse comme une grâce qui passe. L'oeuvre artistique et religieuse d'Hugo est inspirée par sa foi, et elle provoque celui qui se sent en quête des vraies valeurs comme aussi celui qui, insatisfait et inquiet, reste assoiffé des vrais biens. Mon rôle, donc ma mission, c'est de témoigner de la vie de foi et d'amour du frère Hugo, transmettre son message. Son ầme est là̀... et ils sont très nombreux ceux qui le palpents.

\section{Des personnes}

Ces trois interventions restituent et révèlent les composantes qui articulent le dialogue et favorisent la transmission du message. Le schéma des éléments interactifs est présenté au tableau de la page suivante.

Il s'agit donc bien du dialogue entre deux personnes, l'artiste et le visiteur gräce à la médiation de l'objet qui transmet le message. En définitive, ily a bien trois histoires: celle du créateur de l'objet, celle de l'objet lui-même-depuis sa naissance jusqu'à son aboutissement au musće - et enfin, celle que le visiteur colporte avec lui-même.

\section{Une population}

De plus, la démarche ethnologique de A. Neuberg rejoint celle de J. Liebin. Le premier recherche les témoins privilégiếs qui ont bénéficié de l'usage de l'objet et qui en comprenaient le sens: les contemporains du créateur ou les descendants de celui-ci. Lesecond aide une population qui a hérité, malgré elle, de l'objet - au risque de le perdre par ignorance et désintérêt - à redécouvrir la valeur de celui-ci, le message qu'il a à lui transmettre, les bénéfices personnels et collectifs qu'elle peut retirer de son utilisation et enfin, la part que celui-ci apporte dans la construction culturelle et spirituelle de son identité.

\section{Un environnement}

En outre, l'histoire et le message deviennent incompréhensibles et énigmatiques de même que l'objet à son tour inintelligible, inefficace et inutile... si l'environnement social, économique, culturel et spirituel du fabriquant - artisan, créateur et artiste - n'est pas reconstitué et communiqué. Celui-ci, incarné et engagé dans son époque - même s'il est en réaction contre elle - vibre intensément aux courants, aux sensibilités, aux enjeux et aux utopies que celle-civéhicule. En conséquence, il charge inconsciemment et volontairement son oeuvre de ses émotions du moment, de ses drames intérieurs, de son amour du beau travail; de plus, il la pétrit de ses aspirations, de ses raisons de vivre, de sa foi et de son amour des autres - la communauté de ses frères - auxquels la première est destinée. 


\section{Personne}

Objet

Personne

L'artiste

médium symbolique

le visiteur

Emetteur

message

récepteur

Contexte

historique lieu de

présentation histoire

personnelle

\section{Un intermédiaire}

C'est ici qu'intervient dans le dialogue une nouvelle personne, un médium dans tous les sens du terme puisqu'il met des êtres spirituels en communication que les Américains du Nord appellent un «interprètes et les francophones un *animateur». Sa sensibilité, ses informations, ses options personnelles, sa capacité de recueillir le courant symbolique vont favoriser ou non la transmission plus ou moins authentiquedu message et l'intensité plus ou moins profonde et intime de la communication entre le créateur et le visiteur.

\section{Un lieu}

Mais les qualités et la volonté que le guideanimateur implique dans son rồle d'intermédiaire ne suffisent pas. Michel Teheux nous met sur la piste: le lieu où se présente l'objetet l'anbiance quiy règne provoquent un déclic, une étincelle, un effet magique!

Les églises, dit-il, sont des whauts lieux spirituels: c'est-à-dire qu'ils portent en eux-mêmes une dimension quiles dépasse: ils ont une âme... génie du lieu qui s'éveille quand son animateur permet de faire sortir des pierres son intériorité. Des pierres qui parlent parce qu'on les fait parler $\%$. N'explique-t-il pas comment *la grâce de telles expositions (d'artreligieux) naît del'alliance entre les oeuvres exposées et le lieu qui les accueille».

En effet, «le lieu trouve un surplus de sens qu'il n'a pas en lui-même, aussi prestigieux soit-il: en dialogue avec l'édifice, ces expositions explicitent le message latent des prières.

En contre-partie, présentées en de tels lieux privilégiés, les pièces sortent en quelquesorte de leur réserve pour donner à woir et à entendre un mexage jusque là inentendu, une dimension qu'aucun musée, aussi beau soit-il, ne pourra leur donner».

Cette conviction fondamentale des Septennales de Huy est à la base de leur expérience: \&faire dialoguer un espace et ce qui y est présenté et donc donner à entendre une parole que ni l'espace en lui-même, ni l'exposition en elle-même, ne peuvent proférer».

Cela correspond à la philosophie et à la pratique des écomusées depuis 20 ans: plutôt que de déraciner des objets de leur lieu de naissance et de production ou d'usage et d'exercice... pour les émigrer dans des locaux impersonnels et interchangeables, ils les ramènent au contraire dans leurs locaux d'origine: moulin, école, verrerie, ferme, église ou monastère...

Jacques Liébin n'annonce-t-il pas dans son bulletin d'information de l'écomusée régional du Centre sque l'ancienne chapelle dela Grande-Louvière accueillera en 1993, une exposition permanente sur la vie des paroisses de l'Ancien Régimes. En 1988 déjà, suite à l'inquiétude face à l'avenir du mobilier religieux d'églises et chapelles rurales, trop souvent ignoré et abandonné par les responsables locaux, le doyen de la tégion Centre-Soignies avant lancé un appel: *Quel avenir pour le patrimoine religieux?». Une exposition mise sur pied en collaboration avec l'écomusée «s'est attachée principalement à évoquer les $r e$ lations entre l'homme et les objets liturgiques et de dévotion. Les objets furent replacés dans leur contexte à l'aide de panneaux expliquant la génétique de l'objet, sa fabrication, son utilisation, sa conservations.

Parallèlement, le colloque sur le même sujet - dont les Actes sont disponibles ${ }^{(9)}$, a formulé «une hypothèse de solution pour trouver un lieu et les moyens de donner vie à ce patrimoine: l'ancien hôpital SaintJuliusà Boussoit, appartenantau patrimoine immobilier de la ville de $\mathrm{La}$ Louvière».

Lemuséeen Piconrue est situé d'ailleurs au coeur du vieux Bastogne, dans l'ancien couvent de Bethléem (XVII siècle), appartenant à l'évêché de Namur et sauve ce bâtiment abandonné en lui réaffectant une fonction religieuse, culturelle et éducative qui prolonge sa vocation première. N'estce pas ce qui crée ce climat et cette ambiance particuliers que le visiteur perçoit dès qu'il pénètre dans le cloître, à l'entrée du musée?

En définitive, le patrimoine religieux contribue à sa maniêre au développement des parcs de loisirs, leur apporte un supplément spécifique de significations et approfonditainsi les finalités socio-culturelles de l'expérience touristique. $f$

\section{Notas}

(1) André Hut, Mutations culturelles at pratiques touristigues, T6oros, vol. 4, no 2, juillet 1995

(2) Léo Moulin, La vie quotidienne des religieux, Éditions Hachette, Paris, 1978.

(3) Les cimetides, Nouvelles du patrimoine, Bruxelles, no 25, févner 1989. Cécilia Vandervelde, La nécropole de Bruxullea, Commission d'Histoire de l'Europe, 1991.

(4) Paysiges of patrimaine, Nouvalles du patrimoine, Bruxelles, no 39. juin 1991. Presservons nos jandins historiques, un concours europeien, Nouvelles du patrimoine, no 47, décembre 1992 .

(5) Foirea et foraine en Wallonie, Musbe de la Vie Wallonne, Editions Mardaga, Liẻge, 1989.

(6) Pobert Lanquar, Les parca de loisirs, chapitre II, Que saisje, no 2577, Editions PUF, Paris, 1991.

(7) André Hut, Tourisme et musées: complémentarités interactives, Tbor os, wol. 11, no 2, jullet, 1992.

(8) Bruno Bettelheim, Paychanalyse des contes de fóe, collection Réponses, A. Laffont, Paris, 1976.

(9) Quel avenir pour le patrimoine religieux?, ACtes du collogue. bcomusée du Centre, La Louvière, 1988. 